\title{
Some Comments on and Concerning Population Forecasts
}

\author{
by TOR HARTMAN
}

Central Statistical Office of Finland, Helsinki

1. In principle, one can think of three main, different ways of making a forecast:

First of all, a start can be made from the trends in demographic components which exercise an influence upon the future population and its composition. In this connection, it is mostly a matter of mortality, nativity, the net emigration and internal migration, with sex and age taken into account. Secondly, some non-demographic variable can be chosen as point of departure. In this case, it can be some economic veriable such as the development of the national income, with the consequent development of the number of places of work, according to the branch of commerce or industry and the geographical area. With such a variable as the starting point, an attempt is made to deduce the internal migratory movement. However, the mortality and nativity must still be forecast, independently of the initial economic variable. The net emigration can here be taken as a residual variable in such a way that if the number of places of work according to the initial economic variable becomes too small in relation population, emigration takes place, with the opposite taking place if a shortage of labour power prevails. Thirdly, with the point of departure being the present situation, and given valuations, such as rapid an increase in the national income as possible one can attempt to find an optimal allocation of the population in geographical areas, with due regard being paid to age, sex and other variables which exercise an influence. Following this, an endeavour is made to fathom out how rapid a change to the optimal situation can occur. In this connection, the demographic components will be deduced from the given objectives. Nevertheless, mortality should be introduced as an independent variable.

These different forms of forecast have their advantages and disadvantages. From a short-term aspect, a forecast based upon consideration of the trends in the demographic components should be the most realistic. If war or other crises of different kinds do not occur, experience has shown that 
the demographic factors of mortality and nativity change very slowly. So far, no experience has been gained in Finland of forecasts which are based upon some non-demographic variable. It is possible that forecasts based upon non-demographic variables are more realistic in the long run than those forecasts based upon trends in the demographic components.

Forecasts made on the assumption of some objective in view for allocation of the population by means of different variables are hardly realistic at all. They can be perceived as objectives which should be aimed at under certain pre-requisites, but from which one must, for various reasons, often deviate. An optimal allocation is a scheme of thought which we can use to see whether the actual development is on the right or wrong lines. When today we calculate an optimal allocation for a point of time which is, say, ten years ahead, conceivably the allocation is no longer optimal in ten years. Forecasts based upon an optimal allocation have not been worked out in Finland. The difficulties encountered in formulating a forecast of this type are quite certainly considerable in nature.

2. One pre-requisite of good population forecasts, irrespective of how they are prepared, however, is that some perception is possessed of what directs human behaviour. The central concepts of any understanding of human behaviour are motive, preference and reason. It could be said that a person is not "pushed" by reasons, but is "pulled" by the dream of attaining a goal. A man can form for himself an order of importance or preference among the aims he wishes to attain, and can weigh the reasons for and reasons against one against the other when it is a matter of choosing the best way to his goal. Dependent upon the situation in which a person finds himself, the same motive can lead to different modes of behaviour. For example, take two persons, both of whom have financial security as aim. One of them has a high income, and the other a low one. They are accordingly in different situations. The one with a low income favours social reforms, as they increase his financial security, and the one with a higher income is opposed to social reforms, as they increase the taxes payable by those in receipt of high incomes, and accordingly diminish his financial security. Moreover, a person's faith and knowledge influence his behaviour. A person who believes that the rain dance can bring rain is certain to engage in a dance of this nature during a severe drought.

3. Let us turn to the consideration of nativity in the light of the concept of motive, preference, reason, situation and knowledge. As is well known, in western societies nativity often varies in such a way that it is relatively high in low-income groups, lower in medium-size income groups, and then again rather high in groups with high incomes.

Presumably, the high nativity in the lower-income groups is partly attributable to lack of knowledge of contraceptives. Furthermore, one can think of a completely rational reason for high nativity among those with 
a low standard of living. Two parents with a low income could think in this way: We can not save anything for our old age. We get an insignificant old age pension. But our children can contribute to our support when we are no longer able to work by reason of age. As a result of low income, one child can give us very little, but, the more children we have, the more contributions we will get, and accordingly the higher our standard will be. It may be pointed out in parentheses that these motives and reasons probably play a role in those under-developed countries where the old age pension is insignificant. Mostly by reason of the low income for children, they must be many in number if they are to be capable of supporting their aged parents. But many children keep the standard of living low in the under-developed countries. A vicious circle.

In the medium-sized income classes, as a rule in the industrial countries people have their pension arranged, and support in old age does not entail any motive for getting children. At the same time, the knowledge of contraceptives is well-spread. The desire to have a tolerable material existence is stronger than the desire to be gladdened at many children. These motives and knowledge lead to a smaller number of children than in the lower income groups.

In the highest income groups, on the other hand, people have often achieved the material standard they desire, and there is money to spare for the support of more children than in the medium-size income group. It could be said that in this income group people, instead of getting a third car acquire a third child (see scheme below).

Hypothetical scheme of the factors which increase $(+)$ and diminish (-) the propensity to have many children

\section{Factor}

Lack of knowledge/

knowledge of

contraceptives

Financial benefit

derived from many

children

Desire to have

children

Capacity to afford

many children

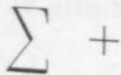

Low income

Medium

income
High income

rar


If the speculations referred to above are correct - and this can be confirmed by means of interviews and other investigations - it can be assumed that nativity will diminish in the lowest income groups, when knowledge of contraceptives spreads, and the motive for many children falls away as the social standard rises, and that nativity will increase in the medium-income groups as incomes rise. It is apparent that people want to have children if they possess the possibilities of doing so.

In a corresponding way, the concepts of motive, reason, and so on, can be employed for such examinations as that of the migration movement. However, mortality has no more than an indirect relation with our motives and preferences.

4. The most recent official population forecast in Finland is a regional one, covering 16 so-called statistical regions divided into the groups of towns and rural communes respectively. For migration movements there have been calculated so-called migration coefficients. The forecast was made in the following way. Let $\mathrm{A}_{\mathrm{x}}^{t}$ denote a population in the age group $\mathrm{x}$ at a time $t$. Let $\mathrm{p}_{\mathrm{x}}{ }^{\mathrm{t}, \mathrm{t}+\mathrm{a}}$ denote the survival number for the age group $x$ between the times $t$ and $t+a$. Further, let $A_{x+a}^{t+a}$ denote the same population $\mathrm{A}_{\mathrm{x}}^{\mathrm{t}}$ a years later.

Then

$$
A_{x}^{t} p_{x}^{t, t+a} m_{x}^{t+a}=A_{x+a}^{t+a}
$$

where $m_{x}{ }^{t+a}$ is the coefficient needed for the similarity to apply if the migration has occurred. The number $\mathrm{m}_{\mathrm{x}}$ has been calculated on the basis of data from the 1950's. The $\mathrm{m}_{\mathrm{x}}$ number is clearly greater than 1 if the immigration is greater than the emigration, and less than 1 if the emigration is greater than the immigration. For the case in which the $\mathrm{m}_{\mathrm{x}}$ number is greater than 1 , the following calculation has been made:

$$
\frac{A_{x+a}^{t+a}-A_{x}^{t} p_{x}^{t, t+a}}{\Sigma\left(A_{x+a}^{t+a}-A_{x}^{t} p_{x}{ }^{t+a}\right)}=k_{x}
$$

(The summation in the denominator has been effected in respect of all regions and types of commune)

In other words, $\mathrm{k}_{\mathrm{x}}$ denotes the immigration proportion obtained by a certain population from the emigration of the populations whose $\mathrm{m}_{\mathrm{x}}$ number is less than 1. Otherwise, the assumptions for the forecast were as follows.

a) The net migration has been assumed to remain at the level which could be registered during the 1950 's, or 7,000 a year.

b) The migratory movement between the regions, and between rural communes on the one hand, and towns and townships on the other, has been assumed with respect to direction and relative intensity to remain at the level calculated for the second half of the 1950's. 
c) It is assumed that the number of survivors remains unchanged during the period of forecast, or as calculated on the basis of the mortality in 1961 .

d) The assumption in regard to nativity leads to a fall in the number of births, and to the differences in the nativity in the various parts of the country being evened out.

The forecast was made in accordance with the folloving formulae:

$$
\begin{array}{ll}
A^{t}{ }_{x} p^{t,}{ }_{x}^{t+5} m_{x}{ }^{t, t+5}=A_{x+5}^{t+5} & m_{x} \leqslant 1 \\
A^{t}{ }_{x} p^{t,}{ }_{x}^{t+5}+k_{x}\left(T_{x}-U_{x}\right)=A_{x+5}^{t+5} & m_{x}>1
\end{array}
$$

where $T_{x}=\Sigma A_{x}^{t} p^{t,{ }_{x}+5}\left(1-m^{t,}{ }_{x}{ }^{t+5}\right)$

and the summation relates to the $\mathrm{x}$-aged populations whose $\mathrm{m}_{\mathrm{x}}$ number is less than 1. In other words, $T_{x}$ denotes the total net emigration from the $\mathrm{x}$-aged populations whose $\mathrm{m}_{\mathrm{x}}$ number is less than 1 . The new fiveyear age groups were dealt with in the same way from the nativity forecast obtained. $\mathrm{U}_{\mathrm{x}}$ denotes the forecast net emigration.

One weakness of the method of calculation described above for the migratory movement is that it takes into account only the flowing out from the emigrant communes, and not the power of attraction of the immigrant communes.

A forecast for the communes in accordance with what is in principle the same method of calculation is being formulated. This forecast is largely to be regarded as an experiment, as in many small communes the stochastic variation of the migration coefficients will conceivably be significant. 